\title{
Performance evaluation of micropropulsion systems with the application of Active Debris Removal
}

\author{
A. Daykin-Iliopoulos ${ }^{1}$ and R. Desai ${ }^{2}$ \\ Queen Mary University of London, London, E1 4NS, United Kingdom
}

\begin{abstract}
Recent micropropulsion developments have increased the operational capabilities of microsatellites $(<100 \mathrm{~kg})$, to the point where they are being considered for demanding missions such as Active Debris Removal (ADR). This study examines the state-of-the-art micropropulsion options that can be applied to a microsatellite performing a range of orbital manoeuvres such as ADR. To this end a generic system architecture is used for this example scenario with ranging delta-v requirements. A variety of propulsion systems are sized accordingly, and the trade-offs between the differing propulsion technologies evaluated with respect to the power requirements, System-Specific Impulse $\left(I_{S S P}\left[\frac{N s}{k g}\right]\right)$, and technology readiness level (TRL), as well as a 'newly defined' volume driven System-Specific Impulse $\left(I_{V S S P}\left[\frac{N s}{m^{3}}\right]\right)$. It was shown that the high specific impulse (> 2000s) and consequent mass and volume savings of miniaturised Electric Propulsion (EP) systems, such as the miniature ion and colloid thrusters, results in optimal $I_{S S P}$ 's and $I_{V S S P}$ 's for the ADR scenario examined. Moreover an in-depth combined quantitative and qualitative analysis identifies delta-v regions most suited to both EP and Chemical Propulsion (CP) systems, as well as highlighting the inherent differences in the varying technologies with respect to volume as well as mass.
\end{abstract}

\section{Nomenclature}

$I_{S S P} \quad=$ system specific impulse

$I_{V S S P} \quad=$ volume driven system specific impulse

$I_{\text {tot }} \quad=$ total Impulse

$M_{p s} \quad=$ propulsion system mass

$V_{p s} \quad=$ propulsion system volume

$M_{0} \quad=$ initial spacecraft mass

$M_{1} \quad=$ final spacecraft mass

$r_{1}=$ initial orbit radius

$r_{2} \quad=$ final orbit radius

$\mu \quad=$ standard gravitational parameter

$V_{0} \quad=$ initial velocity

$V_{1} \quad=$ final velocity

$\Delta i \quad=$ inclination change

$V_{e} \quad=$ exhaust velocity

\section{Introduction}

$\mathrm{R}$ ECENT micropropulsion developments have increased the operational capabilities of microsatellites $(<100 \mathrm{~kg})$, to the point where they are being considered for demanding missions such as Active Debris Removal (ADR) ${ }^{1}$. Many technological challenges exist in the miniaturisation of propulsion systems for microsatellite applications, including the micromachining ${ }^{2}$ of key components and the effective operation under reduced mass, volume, and power

\footnotetext{
${ }^{1}$ Masters student, SEMS, Queen Mary University of London, a.j.n.daykin-iliopoulos@se10.qmul.ac.uk.

${ }^{2}$ Masters student, SEMS, Queen Mary University of London, r.desai@se10.qmul.ac.uk.
}

American Institute of Aeronautics and Astronautics 
constraints ${ }^{3}$. In order to develop and implement these new technological advancements it is vital to understand and quantify, not only the thruster's capabilities, but also the complete propulsion system's performance characteristics.

Previous efforts to characterise propulsion technologies have focused on calculating theoretical efficiencies ${ }^{4}$ and mass-based performance parameters ${ }^{5}$. Following the fundamental research conducted by Stuhlinger ${ }^{6}$ on the theoretical efficiencies of Electric Propulsion (EP) technology, system parameters such as specific mass and mass-to-thrust ratios have been defined and developed for system and mission based analysis ${ }^{4,5,7}$. One particular method which bears mention is the use of a 'system specific impulse' ${ }^{8}$. This method involves combining the total propulsion system mass and total impulse in order to analyse the total system performance and the delta- $\mathrm{V}$ ranges at which contrasting propulsion systems yield optimal performance. The developmental nature of EP technologies however, cause the results of these analyses to quickly become outdated as advancements are made.

In miniaturised propulsion system designs, a shift in the driving parameters towards volume and power considerations adds further complexity to developing an appropriate methodology for evaluating system performance. A comprehensive review of thruster options applicable to CubeSats was conducted in $2011^{9}$, identifying and summarising the relevant progress in the field and the technological developments in the thrusters themselves. The valves, PPU's, and additional system components were left for future research efforts. It can consequently be seen that while there is significant literature on the developmental status of micropropulsion as a technology, there is a distinct lack of analytical methods whereby micropropulsion systems can be assessed. A system-based performance evaluation of existing state-of-the-art micropropulsion options has therefore not been conducted.

The aim of this study is to perform an evaluation of micropropulsion systems in relation to a cutting edge small satellite mission. In this study Active Debris Removal (ADR) is identified as representing the cutting edge technological capabilities of microsatellites ${ }^{1}$. This study thus examines the state-of-the-art micropropulsion options that can be applied to a generic microsatellite architecture performing an orbital manoeuvre representative of an ADR mission.

The analysis conducted herein begins in Section II by defining a mission scenario relevant to ADR. In Section III the methodology is then outlined whereby the various propulsion systems are assessed. In Section IV the results and discussion is then presented displaying the various micropropulsion system performance characteristics as well as the resultant microsatellite capabilities. Section V then concludes the paper with a summary of the key findings.

\section{Scenario}

With the amount of small satellites launching dramatically increasing ${ }^{10}$ it is envisaged that in the near future swarms of ADR microsatellites could be used to actively dispose of satellites and other objects which have failed to deorbit at the end of their useful life ${ }^{11}$. In this study the best estimate for the mass of an ADR microsatellite is found to be $\sim 43 \mathrm{~kg}$ based on previous work conducted by Richard ${ }^{1}$. A conservative estimate of $50 \mathrm{~kg}$ will therefore be used in this analysis along with a $10 \mathrm{~kg}$ microsatellite as a potential projection of the ADR microsatellite architectures in the coming years, as the required technologies develop. In Table 1 the two top-level satellite architectures are outlined. The satellites are assumed to be approximately cubic with volume and power values approximated from the literature ${ }^{1,12}$.

The specific scenario represented in this analysis is an orbit transfer from the densely populated Sun-Synchronous Orbit (SSO) down to a drop-off altitude which complies with the IADC 25 year rule ${ }^{13}$. An orbit transfer from $720 \mathrm{~km}$

down to an appropriate altitude of $300 \mathrm{~km}$ is thus considered, this value a rough average of previous analyses ${ }^{1,14}$. This orbit transfer yields comparable delta- $\mathrm{v}$ values of $\sim 232 \mathrm{~m} / \mathrm{s}$ for both low thrust transfers and high thrust transfers, as calculated using the Edelbaum and Hohmann transfer equations. This delta-v value will then be increased to represent the removal of multiple debris per mission ${ }^{15}$.

As a space debris removal mission has at the time of this paper not been demonstrated, the ratio of chaser satellites to target debris is unknown and difficult to predict. The debris characteristics will therefore not be included in the analysis. As such the primary propulsion system requirements for the transfer orbits will be studied. The remaining mission phases (such as the capture phase) will not be examined. The results from this generalised microsatellite orbital transfer are therefore intended to be applicable to a wide range of possible ADR scenarios and to a wide range of microsatellite missions beyond ADR.

American Institute of Aeronautics and Astronautics 
Table 1: Satellite architectures

\begin{tabular}{|c|c|c|}
\hline Mass $(\mathrm{kg})$ & Size $\left(\mathrm{m}^{3}\right)$ & Power $(\mathrm{W})$ \\
\hline 50 & 0.035 & 100 \\
\hline 10 & 0.010 & 40 \\
\hline
\end{tabular}

\section{Methodology}

In order for a comprehensive micropropulsion system performance evaluation, all components within the propulsion system have to be analysed and quantified as well as the impact on the wider spacecraft architecture. The full system analysis will thus cover the thruster head, tankage \& feed system, power processing unit (PPU) and neutraliser if present, and any necessary spacecraft adjustments, i.e. larger solar panels.

If the thrust-to-weight ratio of the spacecraft is above $10^{-2} \mathrm{~N} / \mathrm{kg}$ the velocity change, $\Delta V$, is calculated using the Edelbaum approximation ${ }^{16}$ :

$$
\Delta V=\sqrt{V_{0}^{2}+V_{1}^{2}-2 V_{1} V_{0} \cos \left(\frac{\pi}{2} \Delta i\right)}
$$

where $\Delta i$ is the inclination change and $V_{0}$ and $V_{1}$ are the initial and final velocities respectively. Otherwise the delta-v is calculated using the Hohmann approximation ${ }^{17}$ :

$$
\Delta V_{\text {Total }}=\sqrt{\frac{\mu}{r_{1}}}\left(\sqrt{\frac{2 r_{2}}{r_{1}+r_{2}}}-1\right)+\sqrt{\frac{\mu}{r_{2}}}\left(1-\sqrt{\frac{2 r_{1}}{r_{1}+r_{2}}}\right)
$$

where $\mu$ is the standard gravitational parameter, $r_{1}$ and $r_{2}$ are the initial and final orbit radius respectively.

The micropropulsion systems are sized using data representative of each propulsion technology. For increasing delta-v the thruster head and operational components are held as constant and the propellant, tankage and feed system scaled accordingly to the complexity of the system. The system scaling factors are either taken from the literature ${ }^{18,19}$ for the respective propulsion technologies or approximated using information on the propellant density, pressure, taking into account ullage and reserve propellant requirements. The propellant volume will be calculated using the ideal gas law and in the case of supercritical propellants the Peng-Robinson ${ }^{20}$ equation of state are used. The propellant mass is calculated using the Tsiolkovsky rocket equation:

$$
\Delta V=V_{e} \ln \frac{M_{0}}{M_{1}}
$$

where $V_{e}$ is the exhaust velocity, $M_{0}$ and $M_{1}$ is the initial and final spacecraft mass respectively.

The inherent power demands of EP systems results in specific wider spacecraft architecture implications, such as additional solar cells, batteries and larger PPUs. The further mass and volume of these components are sized for the respective propulsion systems. To size the solar panel mass addition, Commercial-Of-The-Shelf (COTS) solar panels from Clyde Space, designed specifically for small satellite applications, are extrapolated and approximate scaling laws used, see Figure 1. The batteries are also sized in relation to the solar cells for each propulsion system. The additional power system volume and mass, necessary to support each EP system, can thus be quantified and will be included in the definition of the propulsion system mass and volumes. It is important at this point to note this method is conservative and will exaggerate the spacecraft implications of the EP systems as it assumes that the systems are operating continually alongside all other satellite instrumentation and therefore specifically requires additional power.

American Institute of Aeronautics and Astronautics 

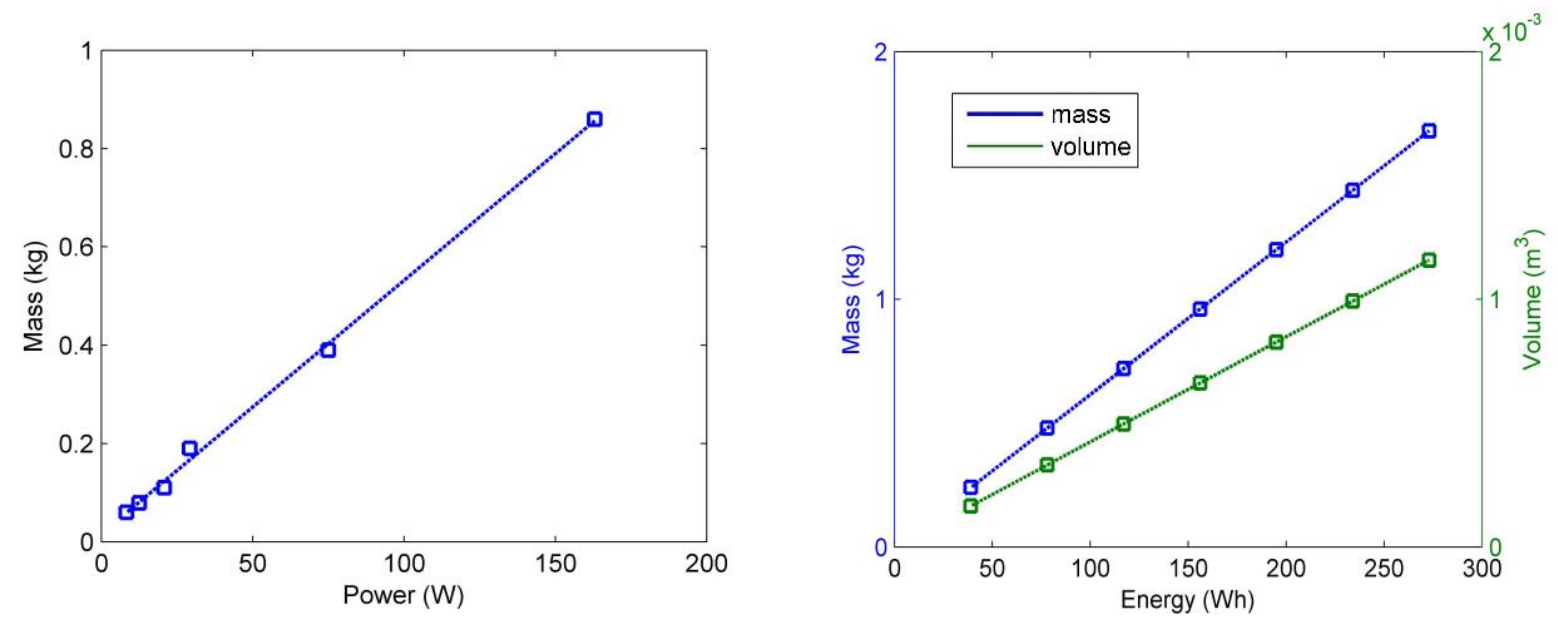

Figure 1 - Scaling of the Power System (a) Solar cell mass is plotted against power (b) Spacecraft battery mass and volume plotted against energy required

\section{Mass driven System Specific Impulse}

The selected propulsion systems will be analysed with respect to mass, power, volume, and TRL as they have been identified as the main driving parameters for microsatellite propulsion systems ${ }^{7}$. The total mass of the varying propulsion systems will be analysed using the 'System Specific Impulse', performance parameter, $I_{S S P}$, as defined by Erichsen $^{8}$ :

$$
I_{S S P}=\frac{I_{t o t}}{M_{p s}}\left\lceil\frac{N s}{k g}\right]
$$

where $I_{t o t}$ is the total impulse required for a given orbital transfer, and $M_{p s}$ is the propulsion system mass.

This performance parameter relates the total impulse delivered for a given mission to the total mass of the propulsion system. The full derivation of this equation with respect to the exhaust velocity and various mass components is outlined in ${ }^{8}$.

\section{New volume driven System Specific Impulse}

Traditionally mass has always been the key driving factor regarding full size satellites due to the high launch costs associated with placing mass into orbit. The standardisation of CubeSat and Microsatellite architectures however mean that volume has become highly relevant. Consequently in this paper a new performance parameter is introduced, the 'volume driven system-specific impulse'. Here the total propulsion system volume, $V_{p s}$, and the total impulse, $I_{t o t}$, are used to quantitatively analyse a propulsion systems performance with respect to volume:

$$
I_{V S S P}=\frac{I_{t o t}}{V_{p s}}\left[\frac{N s}{m^{3}}\right]
$$

This volume based performance parameter is envisaged to be highly relevant for characterising micropropulsion systems.

These two System Specific Impulse performance parameters both scale according to delta-v. Consequently competing propulsion systems performances can be compared in relation to specific scenarios. This, combined with a qualitative analysis of the lifetime/reliability of these emerging technologies, allows for an easy comparison of the key propulsion system characteristics for a given microsatellite mission.

American Institute of Aeronautics and Astronautics 
Micropropulsion is a new and emerging field and as such many of the technologies are still in their early stages of development. The thruster technologies included in this study have been identified as being, or having the potential to be, space qualified within the next few years if subject to an appropriate level of research and development. All of the propulsion systems have been sized to be capable of the impulse requirements of the orbital transfer outlined in Section II and to be in-line with their technological capabilities. The systems are then scaled to be capable of higher delta-vs to investigate the possible removal of multiple debris per mission. It should be noted however that this often assumes the thrusters are capable of lifetimes equivalent to their projected estimates. The sources chosen to be representative of the micropropulsion technologies are shown in Table 2.

Table 2: Chosen characteristic propulsion systems

\begin{tabular}{|c|c|c|c|c|c|c|c|}
\hline $\begin{array}{c}\text { Thruster } \\
\text { technology }\end{array}$ & $\begin{array}{c}\text { Gridded } \\
\text { Ion Engine } \\
\text { (GIE) }\end{array}$ & FEEP & $\begin{array}{c}\text { Colloid } \\
\text { thruster }\end{array}$ & Cold Gas & Resistojet & Monopropellant & Bi-propellant \\
\hline $\begin{array}{c}\text { Company/ } \\
\text { Reference }\end{array}$ & $\begin{array}{c}\text { BUSEK } \\
9\end{array}$ & $\begin{array}{c}\text { ALTA } \\
21,22\end{array}$ & $\begin{array}{c}\text { MicroThrust } \\
23,24\end{array}$ & $\begin{array}{c}\text { Marotta } \\
9\end{array}$ & $\begin{array}{c}\text { SSTL } \\
25\end{array}$ & $\begin{array}{c}\text { Fotec } \\
26\end{array}$ & $\begin{array}{c}\text { Fotec } \\
26\end{array}$ \\
\hline
\end{tabular}

\section{Results and Discussion}

Comparison of the performance characteristics of state-of-the-art micropropulsion systems is not easy due to the emerging nature and developmental status of these technologies. This is caused by the stringent volume, power and mass constraints associated with their implementation. In this section the previously defined mass and volume driven System Specific Impulses are used to quantitatively compare the micropropulsion systems performance in relation to the ADR Microsatellite architectures outlined in Table 1. This can be seen in Figures 2-5, where the $I_{S S P}$ and $I_{V S S P}$ are plotted against delta-v.

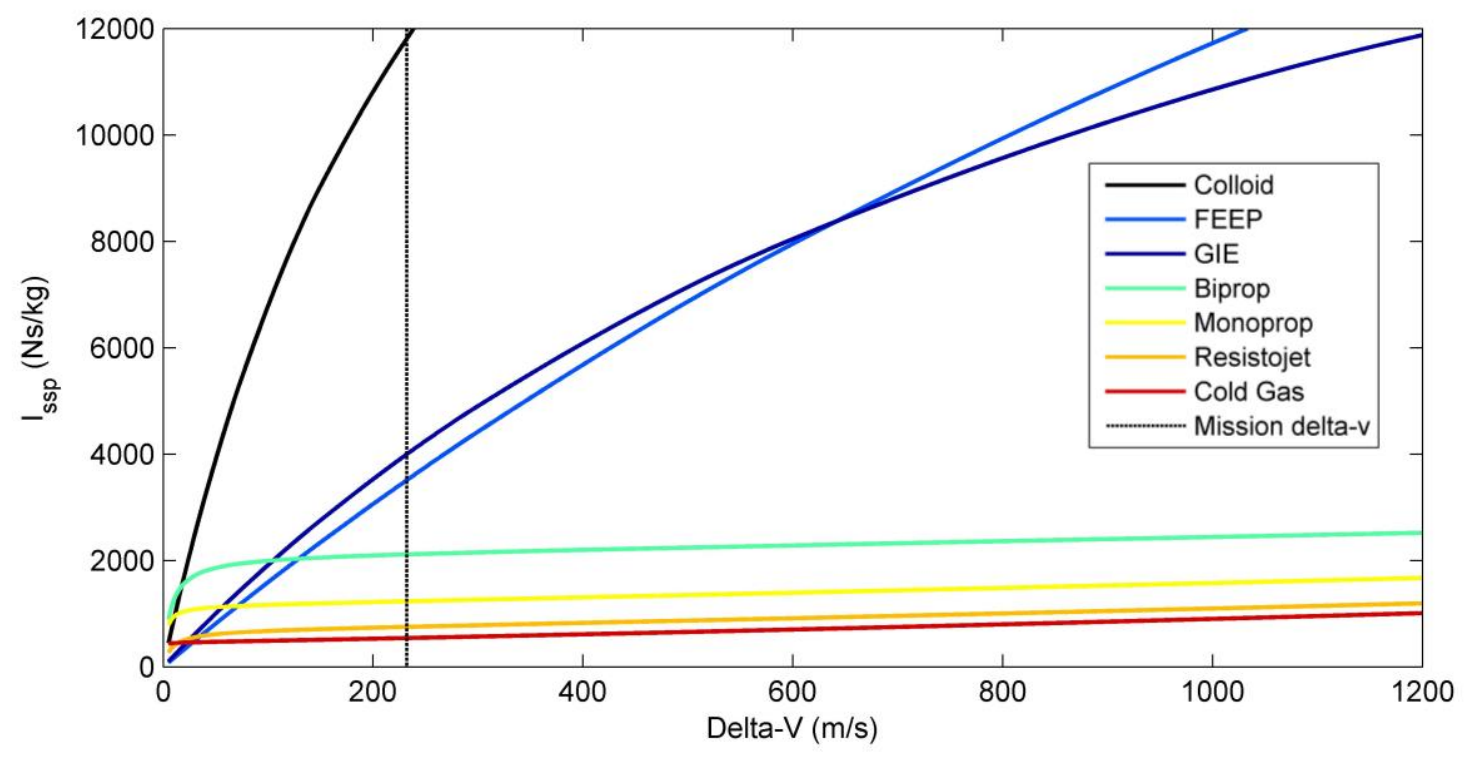

Figure 2 - Mass driven System Specific Impulse plotted against delta-v for the 50kg spacecraft

American Institute of Aeronautics and Astronautics 


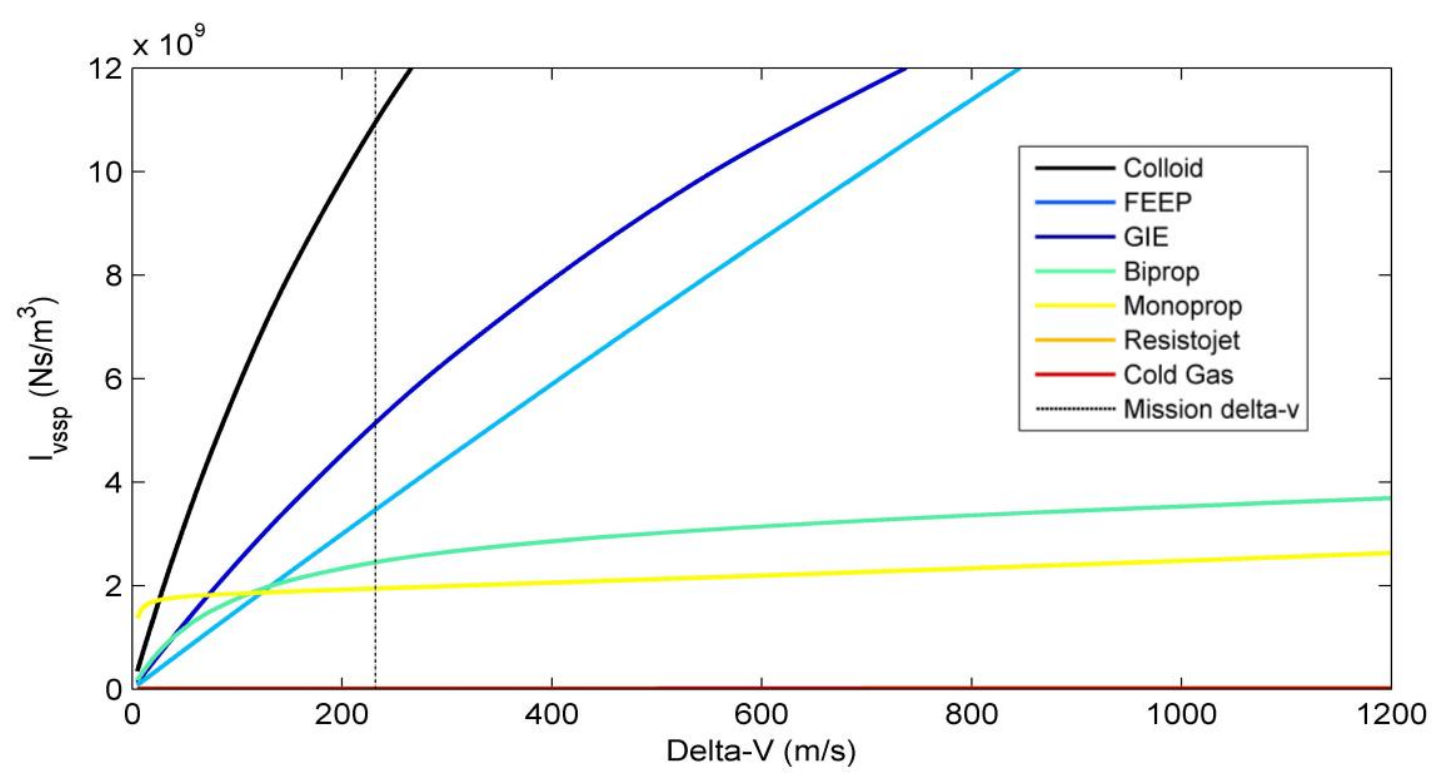

Figure 3 - Volume driven System Specific Impulse plotted against delta-v for the 50kg spacecraft

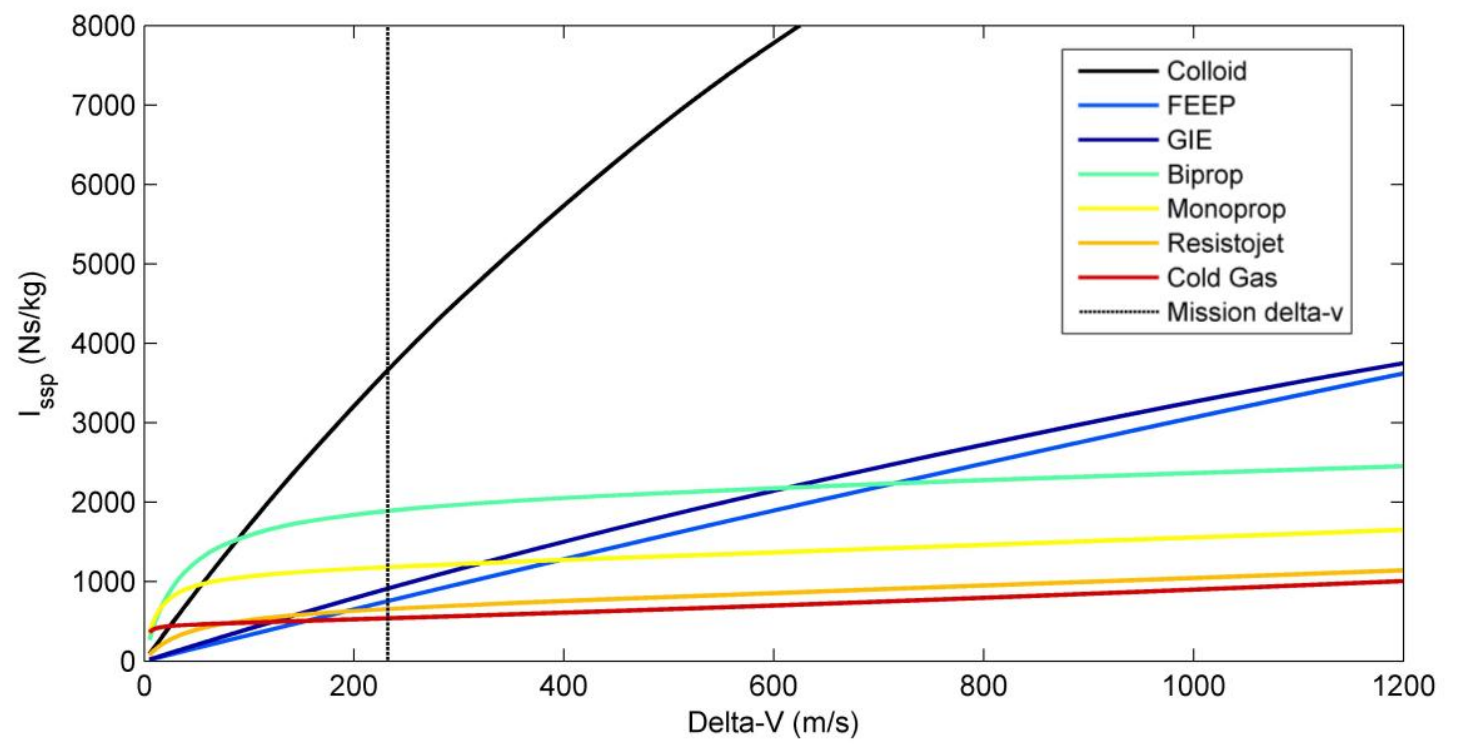

Figure 4 - Mass driven System Specific Impulse plotted against delta-v for the 10kg spacecraft 


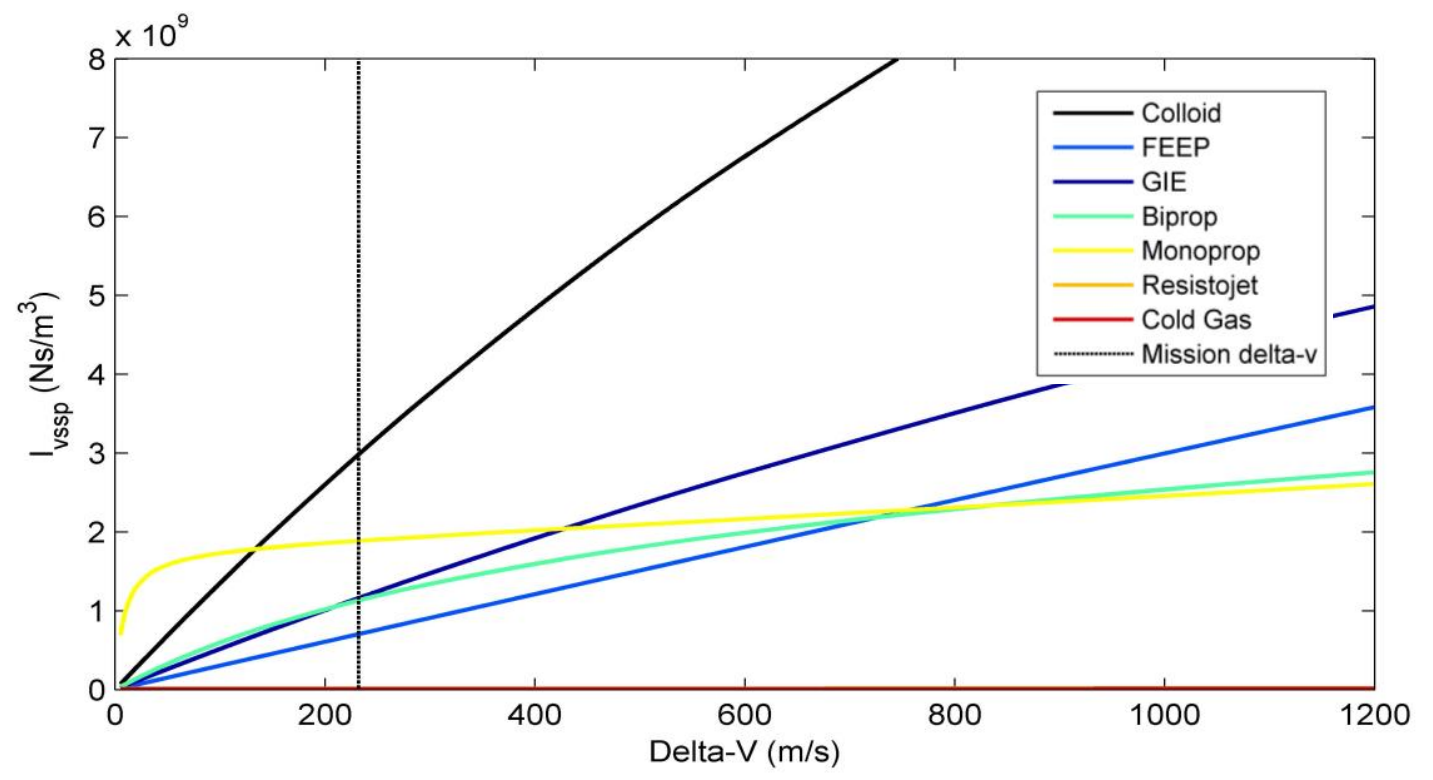

Figure 5 - Volume driven System Specific Impulse plotted against delta-v for the 10kg spacecraft

The differing propulsion technologies can be seen in some cases to have non-uniform trends. There are strong curvatures at low delta-v's $(<100 \mathrm{~m} / \mathrm{s})$ in Figures $2 \& 3$, excluding the Colloid, Gridded Ion Engine (GIE) and Field-Emission Electric Propulsion (FEEP). This is due to the propellant becoming the dominant mass source within the propulsion system as the delta- $v$ increases. Past this characteristic curvature the propellant mass becomes greater than the dry system mass and the increase in the $I_{S S P}$ and $I_{V S S P}$ notably decreases. This is due to the total impulse being a function of propellant mass but not the dry mass. The change in the dominant mass is dependent on the dry mass and Specific Impulse, $\left(I_{s p}\right)$. This is the same for volume, with the addition of the propellant density as a factor. Hence propulsion systems that have low dry system masses and volumes and a high $I_{s p}$ clearly yield better performance in terms of their $I_{S S P}$ and $I_{V S S P}$. The Colloid, FEEP and GIE can be seen not to have this strong initial curvature which can be attributed to their relatively large dry system mass and volume, and high specific impulse. These factors cause the dry system to remain as the dominant mass within the wet system up to relatively much larger delta-vs. These trends are echoed for the $10 \mathrm{~kg}$ scenario, see Figures $4 \& 5$, but are less prevalent due to the reduced total impulse requirement per delta-v.

Figure 2 shows the $I_{S S P}$ plotted for varying delta-vs for the $50 \mathrm{~kg}$ scenario. The Colloid can be seen to perform exceptionally well in relation to the other EP systems due to the low dry system mass and volume. Below delta-vs of $\sim 20 \mathrm{~m} / \mathrm{s}$, the bi-propellant, monopropellant and cold gas systems perform optimally. This is due to the low dry mass and volumes of the $\mathrm{CP}$ systems in comparison to the EP systems. Due to their inherent simplicity and lack of additional system components such as neutralizers and PPUs. Above the delta-v region of $\sim 125 \mathrm{~m} / \mathrm{s}$ the increases in the $I_{S S P}$ are low due to the low $I_{s p}$ 's of the CP systems, and the EP systems can clearly be seen to perform better. This delta- $\mathrm{V}$ region between $20-125 \mathrm{~m} / \mathrm{s}$ can thus be identified as a 'crossover region' over which EP technology starts to perform better than $\mathrm{CP}$ technologies. In Figure 4 the trends can be seen to be exaggerated for the 10kg spacecraft scenario. Here the crossover region is identified as being between $\sim 50 \mathrm{~m} / \mathrm{s}$ and $\sim 400 \mathrm{~m} / \mathrm{s}$. The reason this crossover region is at much higher delta v's is due to the lower total impulse per delta-v. The fact that this crossover delta-v region decreases with increasing spacecraft mass highlights the added value of EP systems vs CP systems for the larger microsatellites.

American Institute of Aeronautics and Astronautics 
In Figure $3 \& 5$ the volume driven System Specific Impulse $I_{V S S P}$, is plotted for both spacecraft. When comparing Figure 2 and 3 there is no real difference apparent in the boundaries of the crossover region although noticeably different trends are evident for some of the thruster technologies. The GIE performs much better in relation to the other propulsion technologies with respect to volume than it does with respect to mass. Conversely the Cold Gas and Resistojet perform exceptionally badly with respect to volume than they do for mass at low delta-vs. For the $10 \mathrm{~kg}$ spacecraft the crossover region is identified at overall similar delta-vs with respect to volume and mass, although once again noticeably different trends exist for several of the thruster technologies. The crossover region here is identified as being between $\sim 100 \mathrm{~m} / \mathrm{s}$ and $750 \mathrm{~m} / \mathrm{s}$.

The reason the monopropellant has favourable characteristics compared with the bipropellant cannot purely be attributed to its simplicity, i.e. lower dry mass and volume. The much lower $I_{s p}$ of this technology indicates that the miniature bi-propellant technology examined in this analysis is at a much lower stage of development.

The micropropulsion systems examined here range from TRL levels of $\sim 4 / 5$ for the Colloid thruster to that of the flight qualified Cold Gas thrusters. While the Colloid thruster performs the best, the low TRL level means development is required. The GIE however has quite a reasonable TRL level and still has reasonable performance characteristics.

As well as the TRL of the propulsion systems, the theoretical lifetime is an issue in attaining the required total impulse. The Field-Emission Electric Propulsion (FEEP) performs well within the performance parameters due to its high $I_{s p}$, but because of the low thrust produced, very high lifetimes are required for high delta-vs and as such it is more suited to high precision applications. The Colloid thruster examined in this study, although operating via a similar electrospray process, has throttling capabilities which results in higher thrust levels with a high $I_{s p}$. The Colloid and miniature GIE have therefore been found to perform exceptionally well in this study for the ADR scenario examined, and also at the larger delta-vs.

Limited surface real estates is also a consideration for microsatellites which is also required for further essential components such as the sensory equipment, solar panels and payloads. The thrust density of micropropulsion systems could therefore also be important when comparing and contrasting competing micropropulsion systems.

These results are for the example scenarios, but can easily be applied when analysing a range of microsatellite missions. The performance parameters used here give a mission specific trade-off in terms of system mass and volume. When deciding on the actual micropropulsion system for a spacecraft, exact mission specific requirements would have to be considered such as thrust accuracy, throttling capabilities, and mission time/risk to mention but a few.

\section{Conclusion}

In this study a range of micropropulsion systems have been analysed and their performance contrasted in relation to a mission scenario representative of an ADR orbital transfer from a densely populated region in LEO down to a drop-off altitude. In the analysis the mass and volume of the entire propulsion systems was quantified and the resultant characteristics of the various propulsion technologies analysed. In the definition of the system all necessary components, such as additional solar panels and batteries for the high power EP systems, were included and the analyses can therefore be considered representative of an initial mission trade-off. The volume based analysis involved

the defining of a new parameter, the volume driven System Specific Impulse, $I_{V S S P}\left[\frac{\mathrm{NS}}{\mathrm{kg}}\right]$. This novel performance parameter was shown to be highly relevant in the emerging field of standardised small satellite architectures. Colloid thrusters and miniature Gridded Ion Engine systems were in particular found to be exceptionally promising candidates for the primary propulsion system on upcoming micromissions. Crossover regions were also identified where EP and $\mathrm{CP}$ each showed better performance characteristics.

\section{Acknowledgments}

The authors wish to thank Professor John Stark, Professor Steve Gabriel, Dr Fariborz Motallebi and the ESA e.Deorbit team for their advice and assistance in undertaking this research. Also many thanks for the funding grants awarded by the School of Engineering and Materials Science, Queen Mary University of London, and the Institute of Mechanical Engineers (ImechE).

American Institute of Aeronautics and Astronautics 


\section{References}

${ }^{1}$ Richard M, Kronig L, Belloni F, Rossi S, Gass V, Araomi S, Gavrilovich I, Shea H, Paccolat, Thiran J. "Uncooperative Rendezvous and Docking.” In 6th International Conference on Recent Advances in Space Technologies, 2013.

${ }^{2}$ Krpoun R, Shea HR. "Integrated out-of-plane nanoelectrospray thruster arrays for spacecraft propulsion." Journal of Micromechanics and Microengineering, Vol. 19, No. 4, 2009.

${ }^{3}$ Mueller J. "Thruster Options for Microspacecraft: A Review and Evaluation of Existing Hardware and Emerging Technologies." In 33rd AIAA/ASME/SAE/ASEE Joint Propulsion Conference and Exhibit, 1997.

${ }^{4}$ Olesun S. "An analytical optimization method for electric propulsion orbit transfer vehicles" In 10th symposium on space nuclear power and propulsion. Vol. 271, pp. 1707-1712, 1993.

${ }^{5}$ Hofer R, Goebel D, Snyder J, Sandler I, "BPT-4000 Hall Thruster Extended Power Throttling Range Characterization for NASA Science Missions” In 31st International Electric Propulsion Conference., 2009.

${ }^{6}$ Stuhlinger, E. "Ion Propulsion for Space Flight.” McGraw-Hill: New York 1964.

${ }^{7}$ Michael M. Micci ADK. "Micropropulsion for Small Spacecraft. American Institute of Aeronautics \& Astronautics.”, 2001.

${ }^{8}$ Erichsen P. "Performance evaluation of spacecraft propulsion systems in relation to mission impulse requirements." In $2^{\text {nd }}$ European Spacecraft Propulsion Conference. Vol., 189, 1997.

${ }^{9}$ Mueller J, Hofer R, Ziemer J. "Survey of propulsion technologies applicable to cubesats”, Jet Propulsion Laboratory, National Aeronautics and Space Administration, Pasadena, CA, 2011.

${ }^{10}$ D. Selva, D. Krejci. "A survey and assessment of the capabilities of Cubesats for Earth observation" Acta Astronautica, Vol. 74, 2012, Pages 50-68.

${ }^{11}$ K. James "Feasibility of microsatellite active debris removal systems" Masters. Dissertation, Aeronautics and Astronautics, Faculty of California Polytechnic Unviersity, San Luis Obispo, CA, 2013.

${ }^{12}$ Catherine C. Venturini, Lyle R. Abramowitz, James D. Johansen, and James G. Gee. "CubeSat Developmental Programs Working with the Community." In AIAA SPACE 2009 Conference \& Exposition, 2009.

${ }^{13}$ IADC, "Space Debris Mitigation Guidelines" Inter-Agency Space Debris Coordination Committee, 2007.

${ }^{14} \mathrm{~F}$. Covello "Application of electrical propulsion for an active debris removal system: a system engineering approach" Advances in Space Research, Vol. 50, pp 918-931, 2012.

${ }^{15}$ A. Ruggiero, P. Pergola, and M. Andrenucci, "Small Electric Propulsion Platform for Active Space Debris Removal” In 33rd International Electric Propulsion Conference, 2013. 1965.

${ }^{16}$ T. Edelbaum "Optimum power-limited orbit transfer in strong gravity fields", AIAA Journal, Vol. 3, No. 5, pp. 921-925,

${ }^{17}$ R.H. Battin, An Introduction to the Mathematics and Methods of Astrodynamics, AIAA, New York, 1987, pp.488-489.

${ }^{18}$ C. Brown, Spacecraft Propulsion, AIAA, New York, 1987.

${ }^{19}$ P. Erichsen, "Spacecraft Propulsion, a Brief Introduction", Computerized Educational Platform Stockholm, 2011.

${ }^{20}$ Peng, D. Y., and Robinson, D. B “A New Two-Constant Equation of State" Industrial and Engineering Chemistry: Fundamentals, Volume 15, no. 1, pp 59-64, 1976.

${ }^{21}$ Marcuccio, S., Pergola, P., Giusti, N., "IL-FEEP: A Simplified, Low Cost Electric Thruster for Micro- and Nano-Satellites", In ESA-CNES Small Satellites Systems and Services Symposium, Slovenia, 2012.

${ }^{22}$ F. Ceccanti,L. Paita, U. Cesari, M. De Tata, N. Giusti, P. Balducci, and M. Del Pistoia "3200 hours Endurance Testing of the Lisa Pathfinder FT-150 Thruster" In 33rd International Electric Propulsion Conference, 2009.

${ }^{23}$ C. Ryan, A. Daykin-Iliopoulos, J. Stark, A. Salaverri, E. Vargas, P. Rangsten, J. Timmerman, M. Jong, R. Visee, L. Vliet, B. Sanders, M. Straathof, F. Nardini, V. Pul-Verboom, S. Dandavino, Ç. Ataman, S. Chakraborty, D. Courtney, F. Belloni, M. Richard and Herbert Shea "The MicroThrust MEMS electrospray thruster: results and conclusions" Space propulsion conference, Cologne 2014.

${ }^{24}$ C. Ryan, A. Daykin-Iliopoulos , J. Stark, A. Salaverri, E. Vargas , P. Rangsten, S. Dandavino, C. Ataman , S. Chakraborty , D. Courtney , H. Shea "Experimental progress towards the MicroThrust MEMS electrospray electric propulsion system", In International Electric propulsion Conference, Washington, 2013.

${ }^{25}$ I. Coxhill, D. Gibbon, M. Drube, "The Evolution of Xenon Resistojet Propulsion Systems at SSTL" In $5^{\text {th }}$ International Spacecraft Propulsion Conference, 2008.

${ }^{26}$ M. Tajmar and C. Scharlemann "Development of Electric and Chemical Microthrusters" International Journal of Aerospace Engineering, Vol. 2011, 2011.

American Institute of Aeronautics and Astronautics 